\title{
Dictionary Production for Census Form Conference
}

\section{R. Allen Wilkinson}

U.S. DEPARTMENT OF COMMERCE Technology Administration National Institute of Standards and Technology

Computer Systems Laboratory Advanced Systems Division Gaithersburg, MD 20899 



\section{Dictionary Production for Census Form Conference}

\section{R. Allen Wilkinson}

U.S. DEPARTMENT OF COMMERCE Technology Administration National Institute of Standards and Technology Computer Systems Laboratory Advanced Systems Division Gaithersburg, MD 20899

April 1993

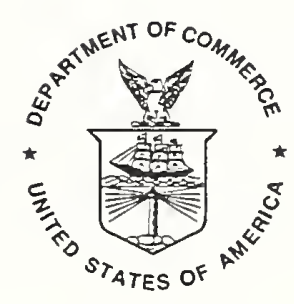

U.S. DEPARTMENT OF COMMERCE Ronald H. Brown, Secretary

NATIONAL INSTITUTE OF STANDARDS AND TECHNOLOGY

Raymond G. Kammer, Acting Director 



\title{
Dictionary Production for Census Form Conference
}

\author{
R. Allen Wilkinson \\ National Institute of Standards and Technology, \\ Gaithersburg, MD 20899
}

\begin{abstract}
There are two categories of data from which dictionaries can be produced. One uses old data or data from a previous collection and the other uses new data or data from a current collection. The old data creates dictionaries that can be used for possible answer examples, assisting optical character recognition (OCR) systems, and training of recognition systems. The new data is the most useful in testing and scoring system results.

For each of the categories above there are two types of dictionaries. These types may be useful for work with the Second Census OCR Conference. The first contains all words that have occurred in the data set being used. For this experimental work, the data set is from the 1980 Census. These words can be misspellings, abbreviations, or correctly spelled words. This first or essential dictionary is easier to create and will not increase the errors which exist in the original data. This dictionary contains all the misspellings, abbreviations and other errors that occur when the original data was keyed from the original paper questionaires. This will make the dictionary useful in describing potential contents of a form set. The second dictionary can be built from the essential dictionary. The second dictionary is one which has the misspellings corrected, the abbreviations expanded, and all the words stemmed into logical minimal stems. A mapping between the essential dictionary to the second or exploratory dictionary is required. The exploratory dictionary is harder to create and may produce more errors than it corrects. This dictionary needs human assistance to be created since most of the steps can not be fully automated. It is also useful in showing a comprehensive list of the possible entries in a form set from previous data, in our case the 1980 Census.

Short and long dictionaries can be produced from both of these dictionaries. The long dictionary contains all of the data from the original Census data, while the short dictionary contains only the phrases and possibly words which occur more than once. The short phrase dictionaries are approximately $16 \%$ of the length of the long phrase dictionaries. They also contain $60 \%$ to $70 \%$ of the phrases in the original sample of 132247 phrases. The short word dictionaries are approximately $45 \%$ the size of the long word dictionaries. About $95 \%$ of the words found in the long phrase dictionaries are contained in these short word dictionaries.
\end{abstract}




\section{Introduction}

In May 1992, NIST hosted the First Census Optical Character Recognition (OCR) Conference. This conference tested the state of the art in OCR technology for isolated hand printed characters. The Second Census OCR Conference is going to address the issue of OCR on form images. The form images being used are "mini-forms". These "mini-forms" will be the Industry and Occupation sections of the 1990 Long Census forms, specifically questions $28 \mathrm{~b}, 28 \mathrm{c}, 29 \mathrm{a}$, and 29b of Form D-2. Each participant will be asked to recognize the hand printed entries for questions $28 \mathrm{~b}, 29 \mathrm{a}$, and $29 \mathrm{~b}$ and return these results for scoring. The ability to use context to assist in the recognition process makes it desirable to produce dictionaries that contain the words or phrases likely to appear as entries.

There are two categories of dictionaries. The category depends on the type of data used to create the dictionary. The first category uses the data from the previous Census, 1980 , and the resulting dictionaries will be used to give examples of possible answers and assisting OCR systems. The second category uses the data from the current Census, 1990. These dictionaries will be used for creating testing data and scoring data.

Within the above categories there are two types of dictionaries that can be produced and might be useful for this conference. The first contains word or phrase answers from similar questions in the Census data. These words include misspellings and abbreviations, as well as correctly spelled words. This type of dictionary is essential for scoring at the character level. This dictionary may also prove useful for improving word or phrase level OCR outputs. The second type of dictionary is one which has the misspellings corrected, the abbreviations expanded, and all the words stemmed into logical minimal stems. This type of dictionary can be built from the first type of dictionary. The second type may also be useful for scoring at the word level. A mapping between the essential dictionary and the second or exploratory dictionary is required. This allows for the creation of a new phrase list from the words in the exploratory dictionary. This new list will still represent the data from the original list but will have consistent phrasings.

\section{Producing the Essential or First Dictionary}

The data starts as a key punch operator's view of what was entered in the Industry and Occupation fields of the 1980 or 1990 Long Census Forms. A sample of 132247 keyed responses is used with each form having four lines of Industry and Occupation data. The first line, Line 0 , is a code for the Industry and Occupation information which follows. The other three lines, Line 1, Line 2 and Line 3, are phrases. These phrases need to be processed to produce a dictionary. The process is to change form entries to phrases, then the phrases to words. The final result is a dictionary that contains all the words which occur in the phrases. This dictionary is long and contains many spelling errors and abbreviations. 


\title{
2.1 Turning form entries into 3 different phrases
}

Each form entry has one encoding line and three text lines. The first line contains Census encoding for the data that follows. For the dictionary production it is not important. Line 1 is the response to, "What kind of business or industry was this?", question $28 \mathrm{~b}$. Line 2 is the response to, "What kind of work was this person doing?", question 29 a. Line 3 is the response to, "What were this person's most important activies or duties?", question $29 \mathrm{~b}$. It is easy to see how the three lines can be interrelated but each line is viewed separately in the dictionary creation process. The very small list in Figure 1 will be used to demonstrate the process of dictionary creation. The phrase lists are generated by removing the same line from all forms. For instance, one phrase list will contain all the Line 1 responses. All punctuation is removed from these phrases. Figure 2 lists all the Line 1 phrases for the short sample list with punctuation removed. The list of phrases from the original Census data has many entries that have multiple consecutive spaces. These are converted into one space to clean up the phrase list. This list is then sorted in alphabetical order and all duplicate entries are removed. A small set of real Line 1 phrases from the original 1980 Census entry list are shown in Figure 3. The resulting lists are very large. For Line 1 there are 46593 unique entries, while Line 2 has 46813 and Line 3 has 61384.

\author{
$0 \quad 041412354$ \\ ZNT-OPERATOR \\ OPEATOR \\ OPERATOR \\ $0 \quad 011250259$ \\ GLASS MARUF \\ MANUFACTURING GLASS \\ MANUFACTURING GLASS \\ $0 \begin{array}{lllll}0 & 4 & 1 & 11274\end{array}$ \\ FFED LOOT \\ SELLING CATTLE \\ SUPERVISOR \\ $\begin{array}{lllllll}0 & 0 & 0 & 1 & 391674\end{array}$ \\ LAMPSHADE MARV \\ MAKING LAMPSHADE \\ GIVING \\ 0042910179 \\ BKING JUDGE \\ JUDICAL \\ JUDGE
}

Figure 1: The entries of the demonstration sample list 
BKING JUDGE

FFED LOOT

GLASS MARUF

LAMPSHADE MARV

ZNT OPERATOR

Figure 2: Examples of phrases found as entries from Line 1 of the demonstration sample list after removal of punctuation and sorting

\subsection{Making Phrases into Words}

The conversion of phrase lists into word lists is rather simple. The first step is to remove all entries that might violate the Privacy Act, such as addresses. Then, using UNIX utilities, it is possible to replace all spaces with newlines. This puts each word on a line of its own. The lists are once again sorted in alphabetical order and duplicate entries are removed. The word list for Line 1 has 13745 words, Line 2 has 13879 words, and Line 3 has 16333 words for this experiment. These numbers show an extreme decrease in list size. This can be attributed to the redundancy of words within the phrase lists.

Figure 4 shows the words produced from the phrases in Figure 2. Figure 5 shows a small sampling of the words from the real Line 1 phrase list. Notice both of these examples contain misspelled words. These lists may also contain words which have the same stem. For example, "advisement" and "advising" have the same stem as "advisor", "advisors", and "advisory". That stem is "advise".

\subsection{Problems with the First Dictionary}

The First dictionary is appealing because it significantly reduces the dictionary size compared to the size of the full 1980 Census data list. Unfortunately, there are also problems with this dictionary. The problems include misspellings, abbreviations, and erroneous data entry. The erroneous data entry was noticed because the key punch operator was to enter the word "blank" when a blank field was found. The word "blank" was misspelled in many ways. Another data entry problem is key punch operators may sometimes spell correctly what is misspelled on the original form. These errors will be discussed in section 3 .

\section{Production of Second Dictionary from First}

The second dictionary needs to be produced in order to improve the scoring and reduce errors. The type of scoring used will help determine if the second dictionary is necessary. 


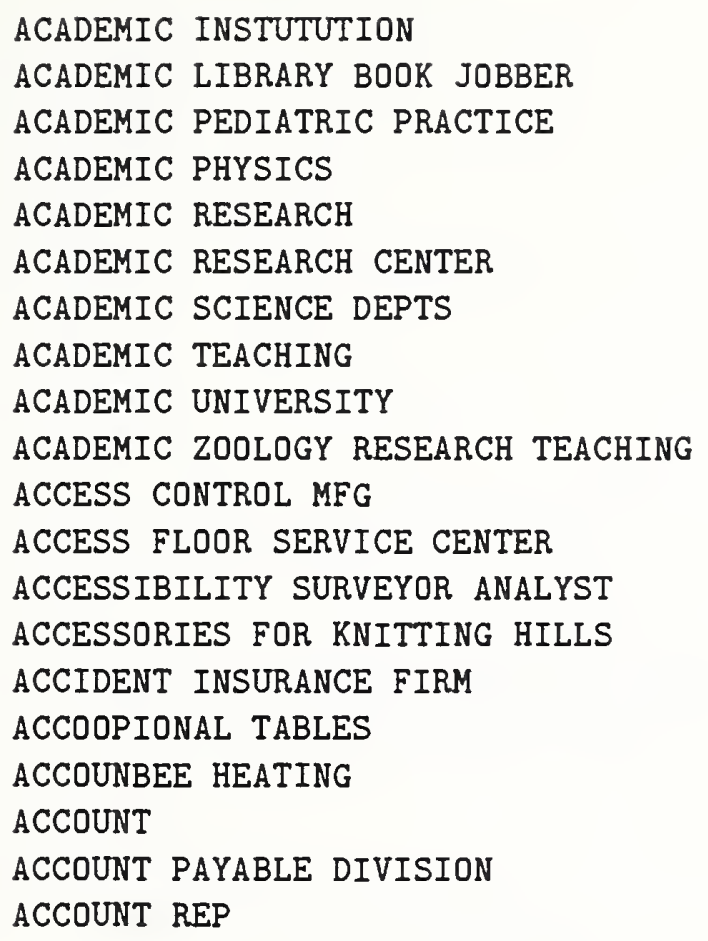

Figure 3: Examples of real phrases found as entries in the 1980 Census sample

Do you score on what exists on the image or by what the writer intended to convey? The best choice is to score the results on what exists on the image because that is what was requested of the data entry personnel. To achieve this, the image must be processed accurately by a human. It is known that this did not happen in all cases with the Census data and therefore the second dictionary is necessary.

One problem is key punch operators misspell words, as mentioned in section 2.3. The word "blank" was to be entered for every field that was blank. Blank is misspelled several possible ways in this data: balnk, blnak, blak, blan, blanks, and blasnk. Therefore, misspellings created by a key punch operator exist. Table 1 shows the occurrence rates of the possible spellings for the word blank. These problems can be solved by creating a correctly spelled dictionary. It must be understood that as soon as you create a correctly spelled dictionary, you can not score by what exists on the image.

Another not so obvious problem is key punch operators can read semantically. Most humans, when entering information into a system, read what was written at the word level. When doing this, some misspelled words are close enough to the proper or correct spelling that humans can recognize the word. The word is then spelled correctly when entered. We have no data about the rate of occurrence of this type of error. This kind of error suggests the use of what the writer intended to convey scoring. To allow for the fact that we do not always know what word was intended with misspellings, the correctly spelled words are also stemmed. The intended word interpretation can be an even greater problem with abbreviations. One added benefit from stemming is the reduction 


\section{LAMPSHADE}

LOOT

MARUF

MARV

OPERATOR

ZNT

Figure 4: Examples of words extracted from phrases of the demonstration sample list

of the dictionary size. This allows for many entries: misspelled, abbreviations, and extensions of the stem, to be mapped to one word. However, this process introduces problems as discussed below.

\subsection{How is the first type of dictionary processed into the second type}

This section explores the issues associated with the production of dictionaries of intended responses. This allows scoring of the word level as an alternative to scoring at the character level where errors in the keyed responses are known to exist. One motivation for producing such a dictionary would be to provide more realistic scores at the word and field level than by simply requiring all characters in a word or field to be correct. It does not appear correct to give the same word and field level scores to "brake manufacturer" and "abrasive manure tower" when the keyed reponse was "brakes manufracturer".

The initial processing of the first type of dictionary is to convert it to lower case. An upper case word will cause strange side effects in the spelling and stemming utilities used. Then the words are spelling corrected and stemmed. These stems are then inserted back into the original phrases to produce correctly spelled and stemmed phrases. These last three steps of the production; correcting spelling, stemming, and making stemmed phrases, need human assistance. This may introduce word level errors while reducing character level errors introduced by the key punch operators.

\subsection{Making words into correctly spelled words}

The word list must be passed through a spell checking program. An operator must look at the flagged words and decide whether to keep the spelling correction or not. Some of the words make no sense. Some words are abbreviations and need to be expanded. 
ADVICORY

ADVISEMENT

ADVISING

ADVISMENT

ADVISOR

ADVISORS

ADVISORY

ADVOCACY

ADVOCATE

ADVRTISING

AENCY

AEOROSPACE

AERATOR

AERESOL

AERIAL

AERO

AEROBIC

AEROCPACE

AERONAUTICAL

AEROPLANE

Figure 5: Examples of words extracted from phrases in the real list of phrases for the 1980 Census sample

Figure 6 shows the misspelled words from the demonstration sample list. Figure 7 shows some of the real misspelled words or abbreviations which need to be corrected. Some words from this list can be corrected while some of them require more information. It is possible to look back at the phrase list to resolve ambiguities. This is part of the reason that mapping between dictionaries, as discussed earlier, must be maintained. Examples of these questionable words are shown in Figure 8 with misspelled words in lower case followed by the phrase in upper case. This figure shows that it may be helpful to look back at the original form entry file, but that even then it may not be possible to resolve all ambiguities. At this point, we have not tried to correct spelling errors, or to expand abbreviations.

bking

ffed

lampshade

maruf

marv

znt

Figure 6: Examples of misspelled entry words of the demonstration sample list 


\begin{tabular}{||l||r|c|r|c||}
\hline \hline Word as found & Occur in & Percentage & Occur as & Percentage \\
Phrase & Occurred & Word & Occurred \\
\hline BLANK & 30457 & $97.469 \%$ & 30499 & $92.539 \%$ \\
BANK & 714 & $02.285 \%$ & 2091 & $06.344 \%$ \\
BALNK & 20 & $00.064 \%$ & 21 & $00.064 \%$ \\
B LANK & 8 & $00.026 \%$ & 8 & $00.024 \%$ \\
BLNAK & 6 & $00.019 \%$ & 6 & $00.018 \%$ \\
BLNK & 4 & $00.013 \%$ & 4 & $00.012 \%$ \\
CLANK & 3 & $00.010 \%$ & 3 & $00.009 \%$ \\
BAND & 2 & $00.006 \%$ & 145 & $00.440 \%$ \\
BLAN & 2 & $00.006 \%$ & 12 & $00.036 \%$ \\
BLASNK & 2 & $00.006 \%$ & 2 & $00.006 \%$ \\
BLAK & 2 & $00.006 \%$ & 2 & $00.006 \%$ \\
BLOCK & 1 & $00.003 \%$ & 136 & $00.413 \%$ \\
BLANKK & 1 & $00.003 \%$ & 1 & $00.003 \%$ \\
BALANK & 1 & $00.003 \%$ & 1 & $00.003 \%$ \\
BLANKB & 1 & $00.003 \%$ & 1 & $00.003 \%$ \\
BLSANK & 1 & $00.003 \%$ & 1 & $00.003 \%$ \\
BLAMK & 1 & $00.003 \%$ & 1 & $00.003 \%$ \\
BLSNK & 1 & $00.003 \%$ & 1 & $00.003 \%$ \\
BNAK & 1 & $00.003 \%$ & 1 & $00.003 \%$ \\
BLANKS & 0 & $00.000 \%$ & 1 & $00.003 \%$ \\
\hline total & 31248 & $100.000 \%$ & 32958 & $100.000 \%$ \\
total misspelled & 791 & $02.531 \%$ & 2459 & $07.461 \%$ \\
\hline
\end{tabular}

Table 1: Table of Occurances for Blank and its misspellings.

\subsection{Making stems}

The UNIX operating system has a spell checker, spell $-x$, that does stemming. Stemming is the removal of suffixes and/or prefixes from a word to produce a shorter word, a stem. The root of a word is the shortest stem possible for that word. A word can have many stems but only one root. In Figure 10, the root for the word "absorbers" is absorb while there are several stems. The appropriate stem must be found and it is not always the root. An operator must decide which stem is appropriate.

Examples of real stemming can be seen in Figure 10, while the stemmings for the demonstration sample list are in Figure 9. When stemming is applied, the word "biomedical" becomes "medic" for a root but the stem "medical" may be more appropriate. Also, "registers" becomes "gist", which is probably not acceptable.

\subsection{Making stemmed phrases}

Stemmed phrases can be made by mapping stems to their correctly spelled words. Then these words are mapped to the word lists. The stem can then be substituted for 


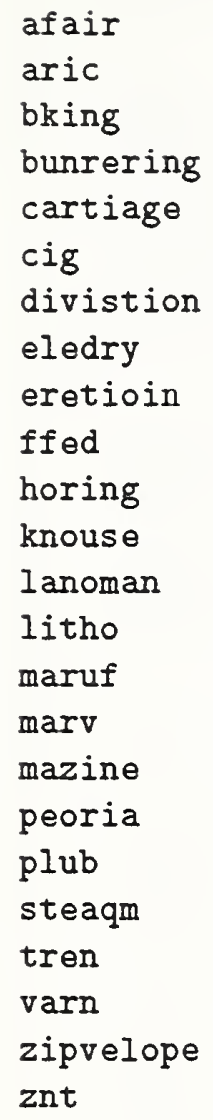

Figure 7: Examples of misspelled entry words

the word in the original phrases. This step also needs human inspection to make sure the new phrases are correct. This step has not been attempted yet.

\section{Other Facts}

All the dictionaries described so far are complete and long dictionaries. This means every word from the original entries is processed and represented in the final result. Short dictionaries can be made which will decrease the length of the word lists. These dictionaries are made by keeping only phrases which occur more than once in the entry lists. The length of the phrase lists then becomes 8216 entries for Line 1, 8516 for Line 2 , and 7831 for Line 3. This is an extreme reduction in the list sizes, while still giving good coverage of the original list of phrases. The lists are $15 \%$ to $20 \%$ of the long list size, but represent $60 \%$ to $70 \%$ of the phrases in the original forms. It also appears that the addresses found in the long lists are not present in the short lists.

The short word lists are generated by using the long phrase list and keeping the words that occur more than once. The sizes are 5950 words for Line 1, 6014 words for Line 2, 


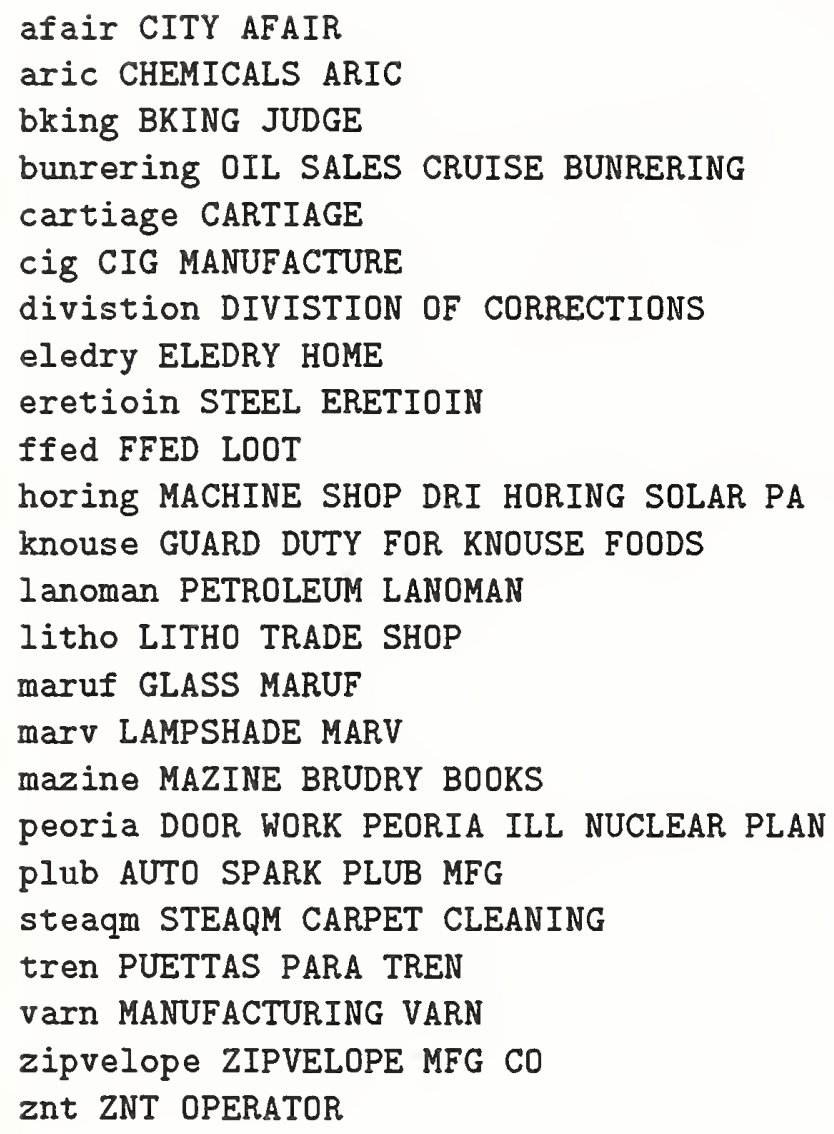

Figure 8: Examples of misspelled entry words and their associated phrases

and 7605 words for Line 3 . This is approximately $45 \%$ of the size of the unique word lists from the long phrase dictionaries. These short word lists contain about $95 \%$ of the words found in the long phrase dictionary. Further investigation must take place before it can be determined if this size reduction will have any adverse effects on the dictionary's usefulness.

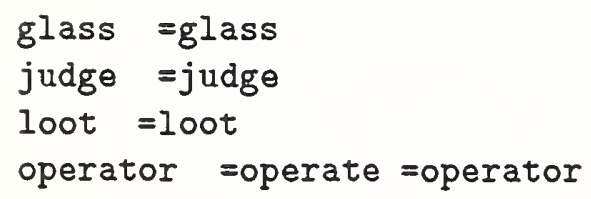

Figure 9: Examples of stemming on the demonstration sample list 


\section{Conclusions}

There are two categories of data from which dictionaries can be produced. One uses old data or data from a previous collection and the other uses new data or data from a current collection. The old data creates dictionaries that can be used for possible answer examples, assisting OCR systems, and training of recognition systems. The new data is most useful in testing and scoring system results.

For each of the catagories above there are two types of dictionaries which can be produced from Census data. The first, the essential dictionary, is easier to create and will not produce more errors than exist in the original data. This dictionary contains all the misspellings, abbreviations and other errors that occurred when the original data was keyed from the images. The essential dictionary is useful in describing the potential content of a form set. It is also useful for scoring at the character level.

The second, the exploratory dictionary, is harder to create and may produce more errors than it corrects. The second dictionary is one which has misspellings corrected, the abbreviations expanded, and all the words stemmed into logical minimal stems. This dictionary needs human assistance to create, since most of the steps can not be fully automated. The exploratory dictionaries are useful for scoring at the word and field level. They would contain only corrected entries that make them much smaller than the essential dictionaries.

Short and long dictionaries can be produced from both of these dictionaries. The long dictionary contains all of the data from the original Census sample data, while the short dictionary contains only the phrases and possibly words that occur more than once. The short phrase dictionaries are approximately $16 \%$ of the length of the long phrase dictionaries, but contain between $60 \%$ and $70 \%$ of the phrases in the original sample of 132247 phrases. The short word dictionaries are approximately $45 \%$ the size of the long word dictionaries. At the same time the short word dictionaries contain about $95 \%$ of the words found in the long phrase dictionary. 


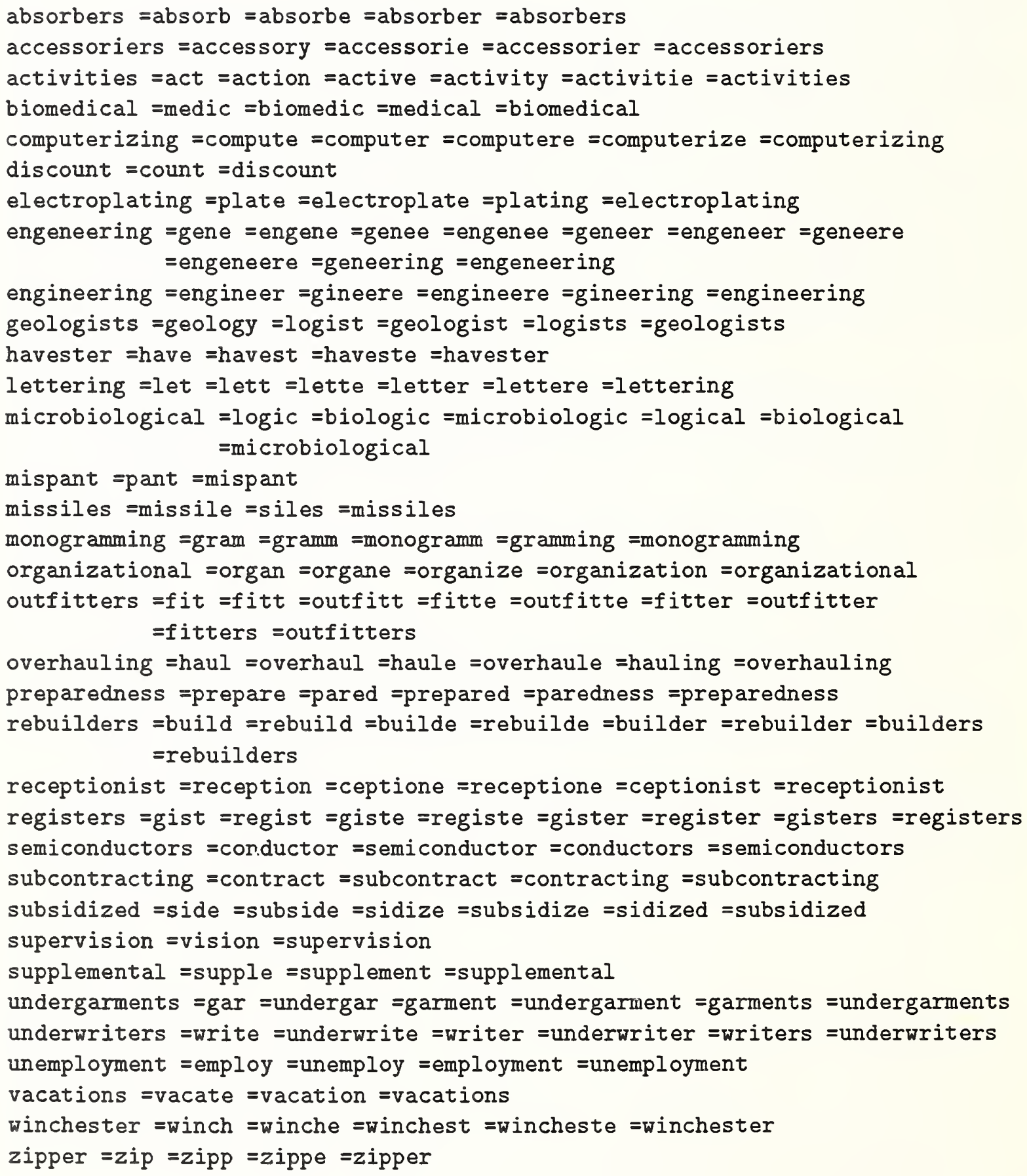

Figure 10: Examples of stemming 

